\title{
Project Teaming in a Democratic Company Context
}

\section{Evangelos Markopoulos}

Department of Business Management, Hult International Business School, London, United Kingdom

University of Vaasa (UVA), School of Technology, Department of Production, FI 65101, Vaasa, FINLAND

evangelos.markopoulos@faculty.hult.edu

\section{Hannu Vanharanta}

University of Vaasa (UVA), School of Technology, Department of Production, FI 65101, Vaasa, FINLAND

Poznan University of Technology (PUT), Faculty of Engineering Management, Poznan, POLAND

\section{hannu@vanharanta.fi}




\title{
Project Teaming in a Democratic Company Context
}

\begin{abstract}
Teaming in democracy generates knowledge, the raw material for innovation, the catalyst for development, progress and extroversion. Democratic teaming, rather than teaming per se, provides people with the space needed to speak and be heard, to give and share, to contribute and to co-evolve in a cooperative and non-competitive way. Democratic teaming belongs to applied philosophy, not applied management, generating added-value for organisations and a shared value for the society. The democratic teaming model is characterized by the principles of placing the right person in the right place at the right time and with the right scope. The model also presents the concept that teams have systemic, dynamic and agile structures, which are affected by the project they are called to serve, within organisationally lean and agile hierarchies for the maximum utilization of employees' intellectual capital. This paper presents the democratic teaming concept and approach within the company democracy model.
\end{abstract}

Keywords: Applied, Philosophy, Democracy, Management, Team, Teaming.

\section{Introduction}

Democracy is a synthetic word, derived from the Hellenic words 'demo' meaning 'the people' and 'crato' meaning 'hold, control, manage'. It is management by the people, the many, not the few (oligarchy), whether a good one (monarchy) or a bad one (autarchy). It is the people - we, the people - who run democracy, structured into teams to achieve progressive evolution and co-evolution, thereby avoiding catastrophic cognitive dissonance, which results into anarchy.

Democratic teaming is not simply a new cooperation practice; rather, it is a knowledge generation philosophy, which produces the raw material for innovation, the catalyst for progress and development, competitiveness and extroversion. It is the effective democratic management and leadership of the people that can generate valid knowledge, as opposed to pseudo-knowledge, which derives from egocentric illusions. On the other 
hand, providing freedom of speech for all can lead to anarchy by those refusing the obligations that democracy imposes, at least on speaking the truth, for the common good. Successful democratic management alone can be a Herculean task without effective team management and leadership.

Teaming is a fundamental democratic management and leadership practice, a fair and honest process for valid knowledge generation among those who have it on behalf of those who need it. Democratic teaming, not teaming per se, provides people with the space needed to speak and be heard, to give and share, to contribute and to co-evolve in a cooperative and non-competitive way [1]. Democratic teaming belongs to applied philosophy, not applied management, generating added-value for organisations and a shared added-value for society [2]. Democratic teaming is a new challenge in modern corporate management and leadership, as people are placed into teams, not only to cooperate, but to think and co-evolve in a cooperative framework of values, ideas and opportunities.

\section{Requirements for the Redefinition of Teams and Teaming for Project Work}

For more than 50 years, most management schools have promoted the concept of teaming as a solution to many organisational problems and as a significant ingredient to organisational well-being and success. There have been hundreds of papers, books and writings on this subject, most of them, if not all, highly theoretical and far from the reality of what it is like running an organisation in practice and dealing with the daily challenges in real-time environments, with real people and under unpredictable circumstances. It is remarkable that, even today, in a fast changing world, there are still experts trying to convince management and leaders with so much platitudinous wisdom - such as managers not being able to do much on their own or without a team [3], or that they should stop thinking of teams as static groups of individuals, who have ample time to practice interaction successfully and efficiently [4] - and with many other recycled management and leadership methods and clichés from the past 50 years at least.

Such points of view indicate that zero progress has been made throughout all these years, or that the teaming concept promises so much that repeating the past can be tolerated. Moreover, there may even be total confusion, if not ignorance, on this subject, which ends 
up with the basics being repackaged as new thoughts and wisdom. It is hard to believe that today there are people who regard teams as static groups; if there are, then this indicates that the teams and teaming, as terms, might be too fuzzy when it comes to understanding and applying them.

There is, therefore, a great need to actually redefine not only the terms of team and teaming, but also the overall framework in which such very important concepts can be applied effectively, realistically and pragmatically. It is time to move on from what shall be done to what can be done, from theory to practice, from illusion to reality, from textbook definitions to industry requirements, and from management announcements to management and leadership actions.

Once we move away from the clichés on team and teaming, which have been repeated politely, but not wisely, for many decades, then these terms might offer something new to the world, the economy, organisations, the people and society. Redefining terms and concepts to fit the needs of industry and society is the only way to make the leap from theory to reality and move forward. One such redefinition regarding teams and teaming can be in the context of project work. People on projects need new ways and cultures in order to work together, while project-oriented teaming can be a new challenge.

\section{There are no Teams, only Projects.}

Teams and teaming represent nothing more than groups of individuals working towards a common goal. It is the need to achieve that common goal that drives the composition of each team to be done with the right people for the right time. Working under the pressure or the need to achieve a goal, especially critical ones, does not leave much room for sentimentality and social relations. Such groups can never be too static nor too dynamic, too small nor too big, too curious nor nonchalant, too weak nor too strong, too passionate nor too otiose, too democratic nor too autarchic, etc. It is the markets, the contracts, the projects, the stakeholders, the risks and many other elements that expect teams to be build based more on knowledge requirements than on social relationships. There are so many elements that need to be analysed prior to establishing a golden rule or piece of wisdom on what teams are and how they should be organized, lead and managed, making it impossible for anything to be achieved via a checklist of team and 
teaming characteristics. It is impossible to define teams and teaming because there are no teams and teaming in modern management, only projects that comprise dynamic teams for their implementation. Once a project is defined properly, it is then easier to define the teams and the teaming process around it. Projects define the teams and not the teams define the projects. Projects are created from the needs of the market, the organization, the industry or the organizational strategy and not by the employees who are called to execute them. Therefore, it is the project the calls for the right teams, dynamically composed in order to be managed well throughout its implementation process.

Teams are dynamic groups of people who can and shall change within a project if needed in terms of the number of members, the type of experts, the commitments, the hierarchy and other criteria dictated by the progress and goals of the project. Projects, on the other hand, are systemic and dynamic, as well as based on the customer, the legislation, the budget and schedule constraints, technology constraints and other issues, which define the project implementation and operations environment.

Therefore, teaming and team management are also very dynamic concepts, which are not so much affected by the people but by the project defining or describing those needed in relation to the team structure, the management process, and team roles and characteristics, both as a group and per individual. This new project-team relationship is not based on the idea that teams are formed to serve projects; rather, projects form the teams to serve them (Figure 1).

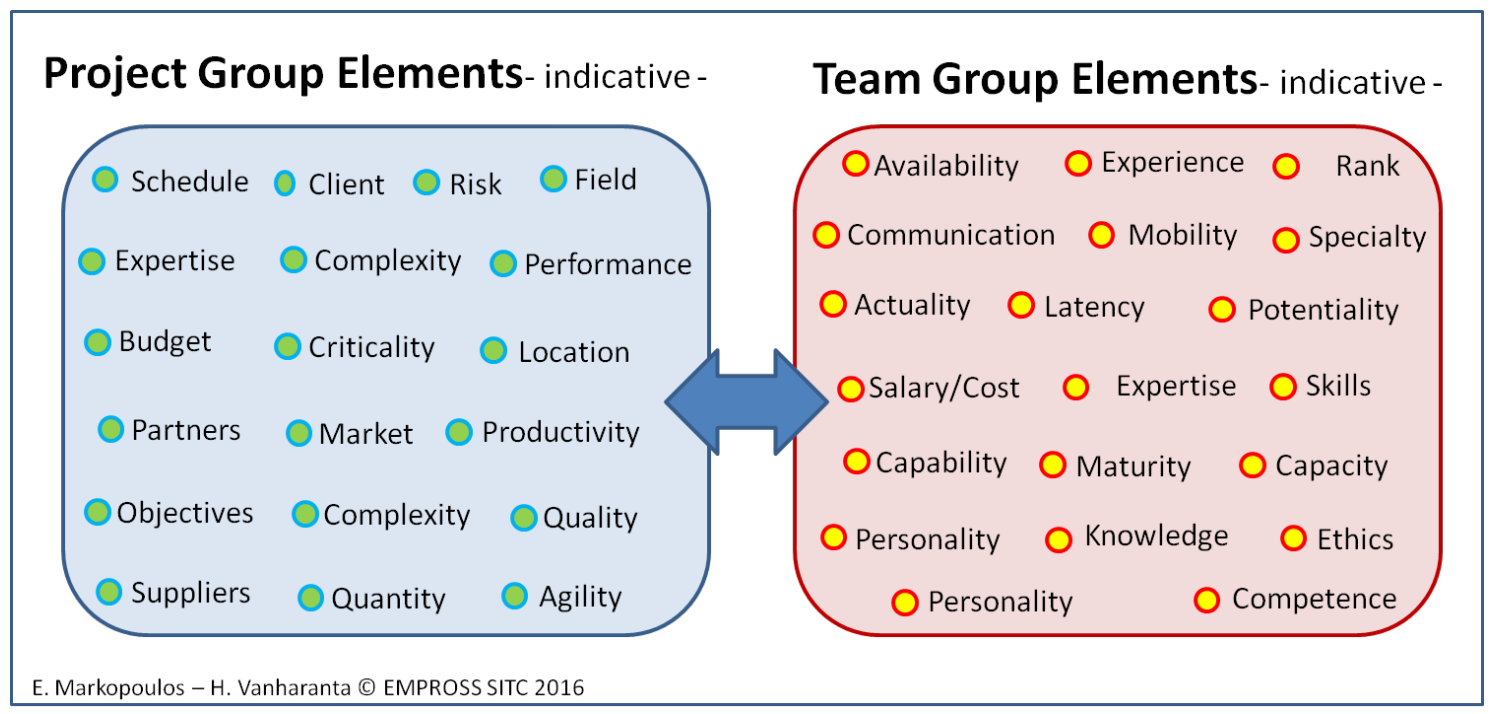

Figure 1. The difference between project and team group elements (indicative) 
In this sense, no team member is permanent and static in a team, while no team is permanently static within an organisation, as projects are driven by the market, the competition, and the organisational objectives, goals and strategies. The team and teaming idea in a project context is that the team group elements (individual and collective) evolve into the project group elements.

Such an achievement can be an important measure of a project team and is normally calculated by money. That said, on many occasions, the most important team group elements with existing resources are actuality, capability and potentiality, which significantly describe the operational side of the team, i.e., latency, productivity and performance [5] .

\section{The Team Builder in a Project Context}

Under the 'project to team' and not the 'team to project' approach, the coordination of the intellectual and human capital of an organisation becomes crucial to its operations and success. This 'project to team' thinking turns all people in an organisation into project implementation assets, for the success of the project, regardless of their position or status in the organisation.

In this sense, the role of the 'team builder' becomes similar to the role of the coach in an athletic club. The coach has to merge team group elements (athletes) with the project group elements (game needs) differently in each game. The coach, as an autonomous authority, is above the players, management, advisers, consultants and experts with supreme powers for the benefit of the club. The team builder should also be above team members, as well as project elements, when this merging happens. The team builder first analyses each project specifically from the organisational dimension and the project implementation dimension. The organisation dimension includes the elements of the project with impact on organisational operations, such as the competition, time to market, innovation, new products or services, and governmental requirements. The project implementation dimension includes the elements of the project with impact on its implementation, such as time, budget, expertise, technology, process and experience. The team builder within the athletic club metaphor is the coach who analyses the scope of the 
game and the game itself, prior to deciding the starting line-up and the substitutions to be made during the game.

Having undertaken such an analysis, the team builder selects the team members for the implementation of the specific project, which is in a similar way to the authority that the coach in the athletic club enjoys when selecting the right team for the right game. The team builder can select team members, not only from those available, but also in terms of taking risks to integrate various types of skills, capabilities, competence, expertise, management levels, seniority, experience levels etc., which seem to be needed in order to form the right team to start the project. An example of the relationships between team builder, project group elements, personnel and implementation team, in which the team builder selects a person for a specific task on a project, is presented in Figure 2.

The team builder, just like the coach, can make any substitutions needed during the implementation of the project, change team members' roles or redesign the implementation strategy. The team builder is the absolute master in management and leadership, as well as the utilization and allocation of human recourses in an organisation, including senior management. If there is a need for the CEO to be part of a team for a specific purpose and role, such as motivation, inspiration and others, the team builder can request that. Likewise, CFOs, COOs or other $\mathrm{C}$-suite executives are all at the command of the team builder to participate in a team when requested. 


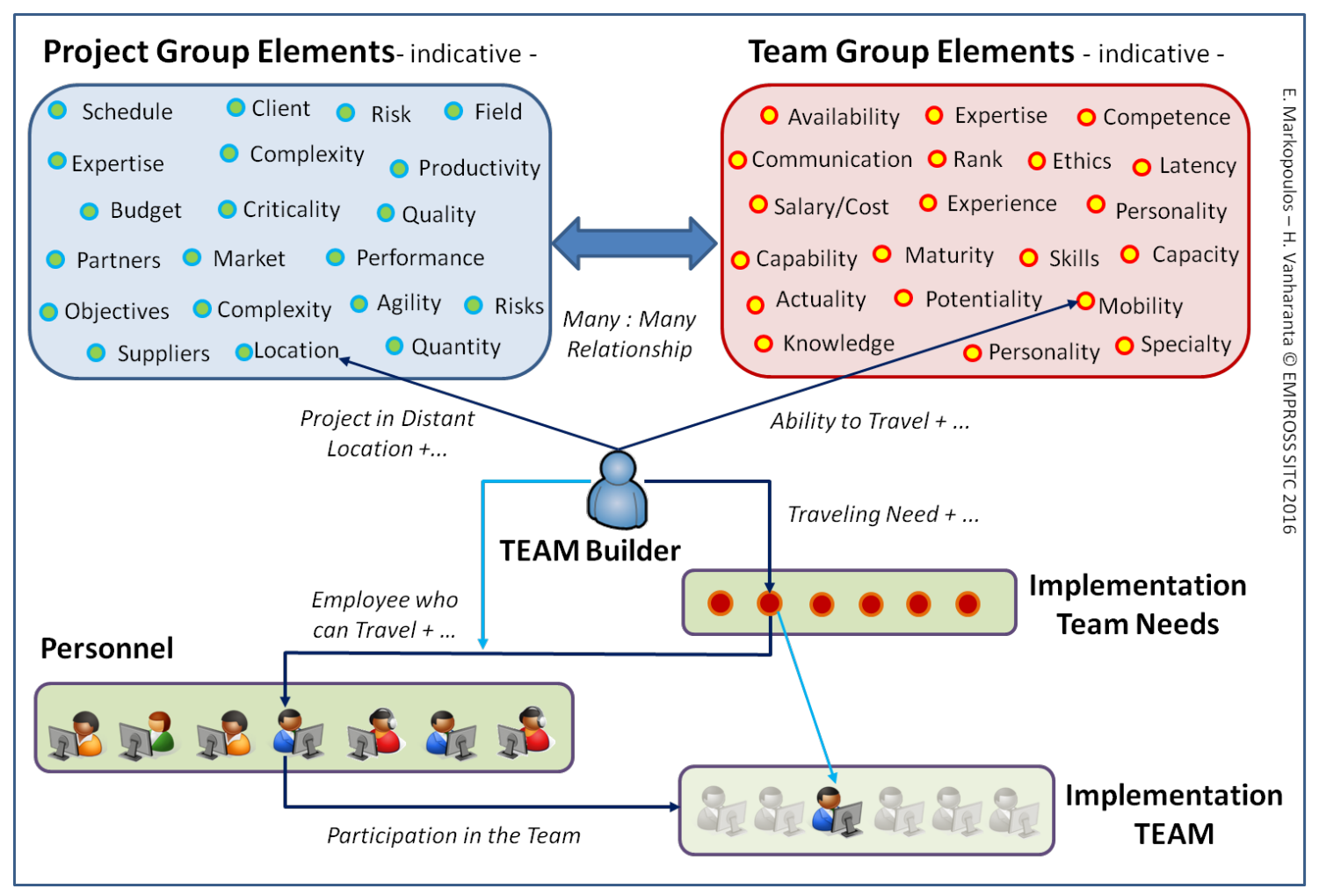

Figure 2. The role of the team builder in the project context

The team builder knows the availability of the resources and takes all necessary risks to promote not only the most capable ones, but also the most talented and most willing to work, or the most innovative, active and enthusiastic, according to the project constraints and requirements. It is the project requirements, criticality and conditions that define the team per project. It is the knowledge and the desire to work exceptionally that is valued more that the social relationships established in an organization or in a project. The project is above all, above the people and above the organization, therefore those who compose the team for the project are the most suitable ones per case. Team building, and teaming in general, call for developing both affective (feeling) and cognitive (thinking) skills among the team group elements [6].

\section{Dynamic Teaming}

Projects can be considered as systemic living entities [7] [8] [9]. Projects change over the course of their development for many reasons, such as new or forgotten requirements, insufficient design, design optimization, timing, budget, schedule and client interference, as well as many other conditions that usually appear more or less in the course of project 
implementation. Therefore, as projects change, so do teams. It is very unlikely for the team starting a project to finish the project, staying unchanged in all implementation stages. The type of each stage and the execution process that need to be followed require variations among the team members in terms of expertise regarding the implementation process, field expertise, communication, technical expertise and others [10].

A very common project implementation approach used when implementing projects with both stable and unstable requirements is the evolutionary model [11]. This approach is based on the idea of rapidly developing an initial project implementation cycle with deliverables from very abstract specifications. This kind of prototype is gradually modified and enriched according to the client's appraisal, requirements and maturity or through an incremental implementation strategy. Each version of the project deliverables inherits the best features from earlier versions. Each version is refined, based upon feedback from the implementation team, the client or the market, in order to produce a new version, which satisfies the new needs. At this point, the project results may be delivered or re-implemented using a more structured approach in order to enhance robustness and maintainability. Specification, development, validation and verification activities are concurrent with strong feedback between each other. Under the logic of the evolutionary implementation model, a teaming evolutionary model can be defined based on the evolution of the implementation team in the implementation process (Figure 3). 


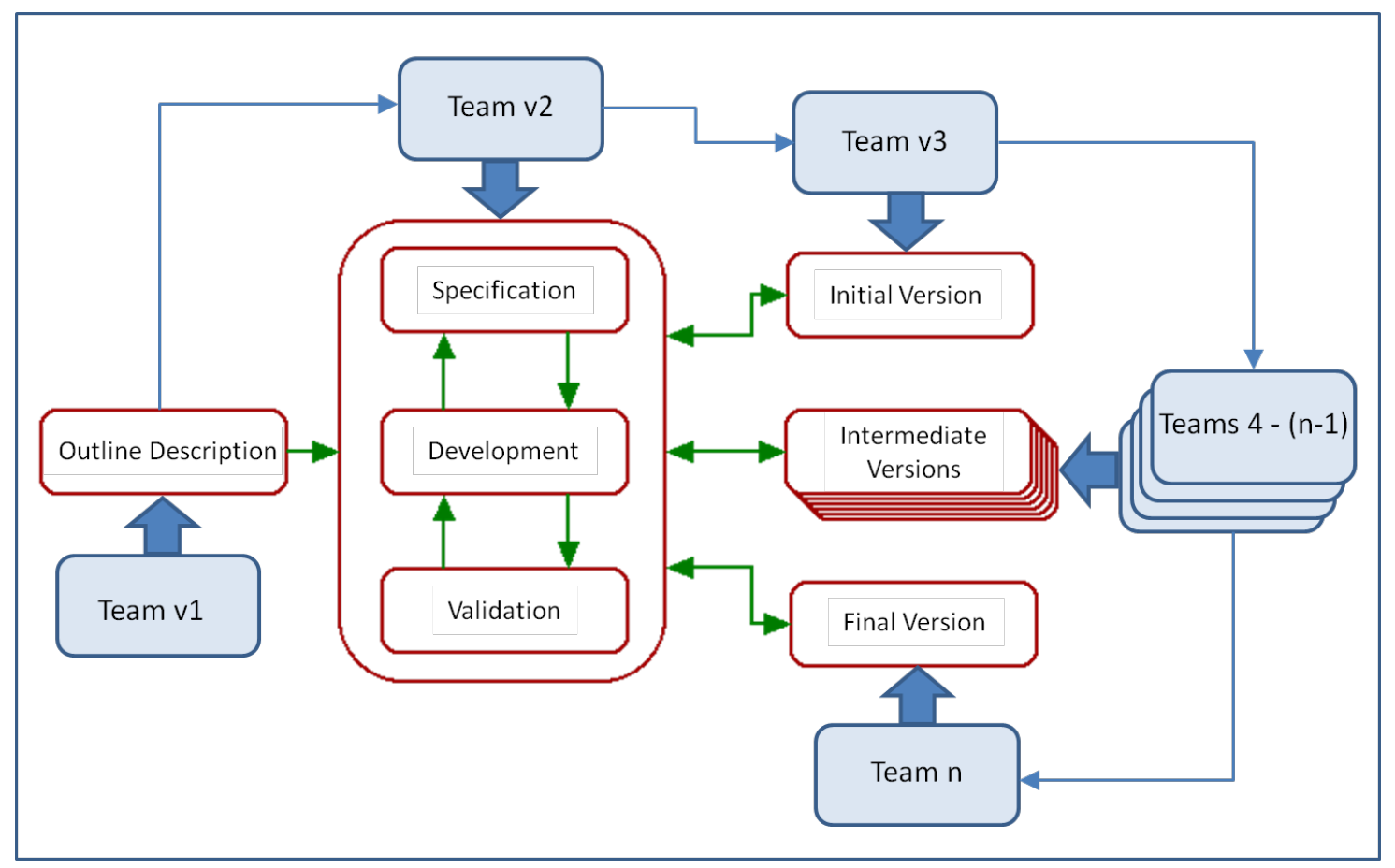

Figure 3. The evolutionary teaming model

The team that initiates the project requires more scheduling expertise, implementation strategy, technical planning, financial planning, communication skills and other skills needed for better understanding and planning of the project. The team that actually implements the project and delivers the first version of the results is a more technical team. The same team, based on the evolution of the project, in response to client involvement, new requirements, test results, optimization, market feedback and other reasons, can generate intermediate versions of the deliverables with the help of business analysts, field experts, domain experts etc. The number of versions is related to the effort in implementing the project's requirements. In this versioning process, the project implementation team can be changed in order to integrate various types of experts needed for the completion of each new version. The final version of the project is the one that closes the project implementation loop (iteration), which requires special expertise, such as project delivery strategy, communication, documentation, prototype deployment, presentation and other primarily non-technical skills.

Regardless the type of project, field, size, duration etc., projects remain the same as they are all characterized by the planning, the execution, the testing and the delivery phases, meaning that teams must be agile in order to complete these phases faster, cheaper and better. Agile teaming is crucial in the effective management of any project. Agility provides the flexibility needed on the project implementation and management process 
to overcome bureaucratic, decision making, management or technical hurdles that could affect the implementation pace of the project or its technical implementation.

\section{Democratic Teaming and Teaming Culture}

The most serious challenge that a team builder has to face is to identify the talents of the people in an organisation. This qualitative analysis is different and more complex than any quantitative one. Building teams quantitatively is quite easy and straightforward, based on the availability of the personnel, as well as their expertise as a match for the project requirements, and generally involves a typical checklist of qualifications. Following this approach has been proven, even at management level, to be the safest way to make decisions, although it can also be a most catastrophic approach for organisations, as much of the talent is lost due to bureaucratic teaming practices. On the other hand, the qualitative team building process includes the risks taken by the team builder on building teams, not only in terms of the qualitative information gathered by the team leader per project, but also in relation to each potential team member's intuition, feelings, values and beliefs.

It takes true leadership to think and stay out of the box when needed, to take the risks and develop a culture that generates winners and doers. It takes a charismatic leader to learn about their people and get the best out of them. It takes power to give power and freedom to all, to speak up and validate. Employees deserve a chance of a career, not just a job, a journey and not a settlement, respect and not for sympathy. Today's leaders must, therefore, build a culture where teaming is expected to feel natural and start by helping everyone to become curious, passionate and empathic [12]. Such leaders must be able to distribute leadership and promote the purpose of teaming in terms of expanding knowledge and expertise, so that organisations and their customers can capture the value $[6]$.

This distribution of power, as well as the freedom for people to speak, have the opportunity to challenge, and share and demonstrate their knowledge and capacities, can best be achieved with the development of a corporate democratic organisational culture. Democracy is a political system, as well as an organisational one, which gives people the opportunity to speak and act freely. It is a system that exposes the quality, capability and maturity of the people thorough the leaders they vote in and the opportunities they receive, for their own benefit, as well as that of their organisations and wider society. A 
democratic corporate culture can provide the team builder, the leader of the leaders in an organisation, the process to achieve 'gnothi seauton', that is, self-awareness in relation to the people, their talents, skills, capability, competence and maturity, which an organisation can gather in order to staff each project accordingly, as well as achieve organisational success through efficient and effective project implementation.

Democracy in business and government contexts in modern societies is a must, as it is the only system that can successfully cope with the changing demands of contemporary civilization [13]. The creation of democratic company cultures has been, for many organisations, an ideal effort in accomplishing harmony in management and leadership. Applying project teaming in a democratic company context means that people are given the space needed to speak and be heard, to give and share, to contribute and to co-evolve in a cooperative framework [1]. Project teaming, in turn, seems to offer the raw material for innovation and knowledge creation; in other words, it is definitely the catalyst for positive progress on projects and in terms of company performance. Such a democratic teaming approach is based on applied philosophy, not applied management, generating added-value for organisations and shared added-value for society, increasing the productivity, profitability and overall performance of the organisation. Democratic team management and leadership in a company democracy framework contributes towards solving chronic enigmas in corporate management and leadership, in terms of what is organisational knowledge, where it is located, how to obtain it, how to utilize it and how to capitalize on it financially and socially.

The democratic project teaming model and concepts, through the company democracy model, can be presented in a systemic way, characterized by the principles of placing the right person in the right place at the right time and with the right scope. The model presents the concepts and characteristics that democratic teams have or should have. From the employee point of view, the main idea is to be given the freedom to speak and participate in project work with the maximum utilization of employees' intellectual capital in order to create organisational added-value.

\section{The Company Democracy Model}


The company democracy model [14] [15] is based on the wisdom of the ancient Hellenic Delphic maxims [16], primarily on 'gnothi seauton' (know thyself), 'metron ariston' (moderation is best) and 'miden agan' (nothing in excess).

The model is executed through a framework in which an organisational evolutionary spiral method is used for the creation and execution of knowledge-based democratic cultures. The co-evolutionary spiral method contributes to the identification and achievement of the capacity, capability, competence and maturity needed to turn data, information and knowledge into innovations. The spiral process, in this context, is based on the idea of the degree of democracy in organisations. The model is structured so that the method reflects co-evolution methodology [17] and its applications in organisational democratic performance [18]. Both co-evolution and the company democracy spiral method aim to create an organisational knowledge-based culture [19]. Both methods utilize organisational knowledge by developing a culture that can constantly contribute to the organisation by transforming tacit organisational knowledge into explicit knowledge [20].

The company democracy spiral method levels form a pyramid structure. The pyramid shape has been chosen to point out the incremental progression of the levels and also to illustrate that not all whom attempt this route can reach the top without real commitment, determination, and organisational capability, competence and maturity (Figure 4). 


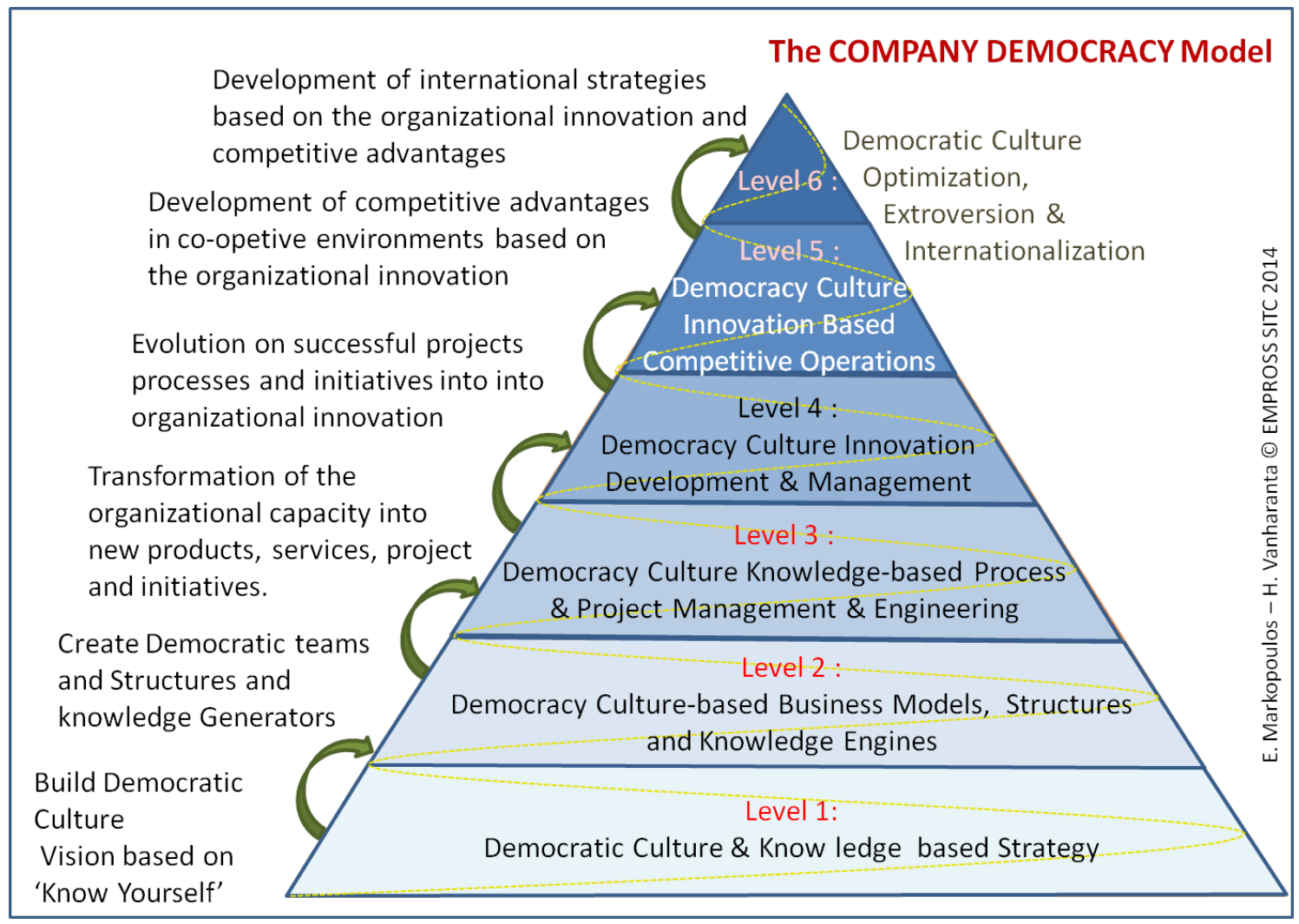

Figure 4. The company democracy model pyramid with structured stages [14]

The company democracy model levels provide the actions to be performed in order to identify the extent of company democracy, based on the individual and collective evolution dimensions (Figure 5). 


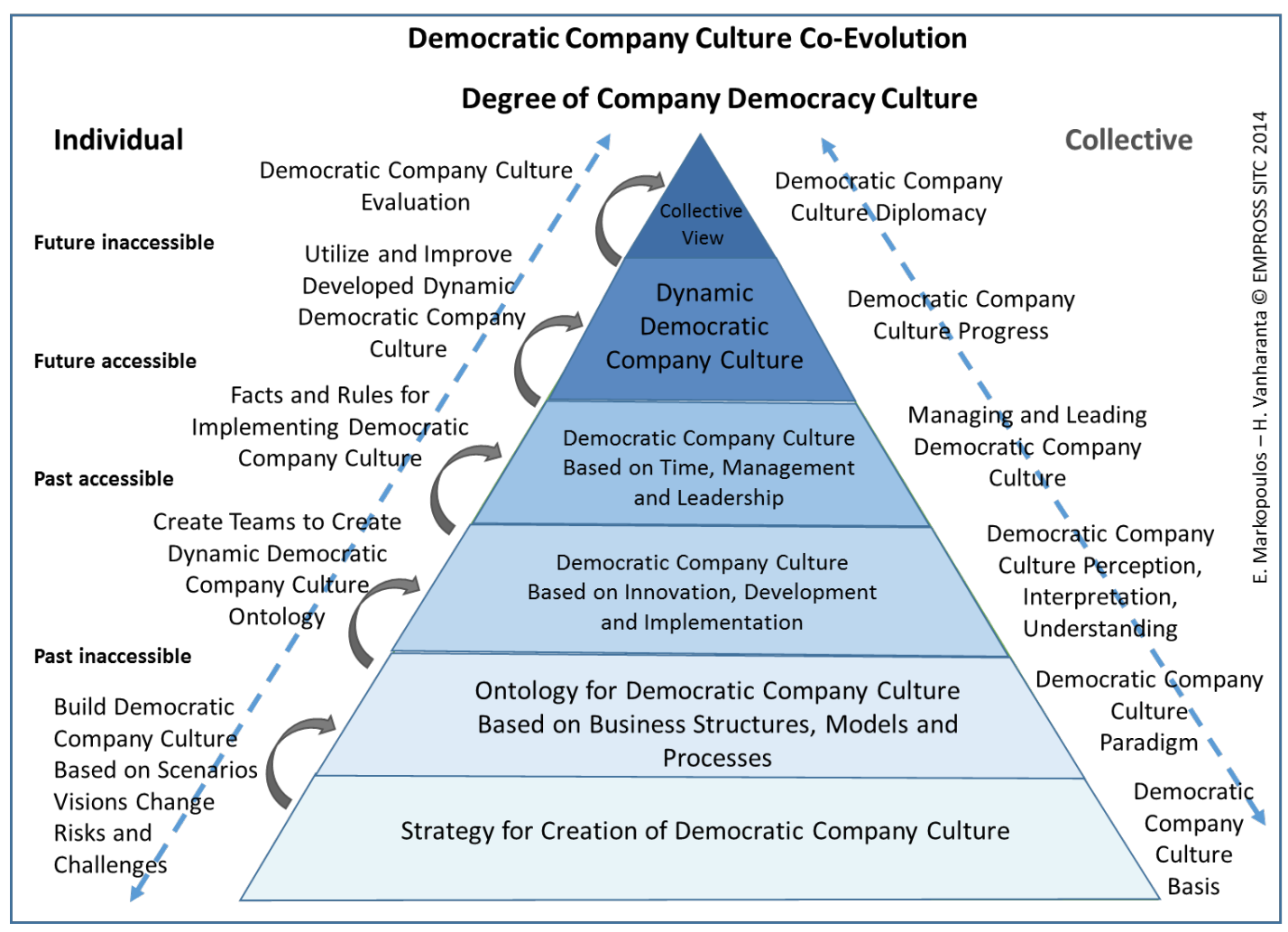

Figure 5. Coevolutionary individual and collective dimensions in dynamic democratic company culture development [14]

The individual side of the pyramid can remember and identify the previously inaccessible part of the company democracy process, so that it is accessible today and in the future. From a collective point of view, the basis of the democratic company culture must be firm, while the created paradigm must contain all the known information and the democratic company culture must be understood, interpreted, and perceived by each member. Therefore, for an organisation, it is important to first understand the current extent of democracy and how this might be extended over time through democratically oriented changes.

The company democracy model provides a structured path to support an organisation to reach democratic management, leadership and operations. As the goal is democracy in terms of innovation, profitability, productivity, performance, competitiveness and extroversion, people must first learn to be cooperative in order to coexist and coevolve. The company democracy model is both systemic and dynamic in nature with characteristics of fuzziness. 


\section{The Company Democracy Teaming Model}

Teaming is a leading element in the company democracy model for management and leadership in an applied philosophical framework, which promotes organisational innovation, competitiveness and extroversion from within. The company democracy model can be affectively and efficiency applied in human recourses and team development and management through the democratization concept it represents. The model provides an organisation with the opportunity to identify the skills, capabilities, competence and maturity of its human resources by simply giving the people a place to stand and participate within a democratic culture.

Teaming through the company democracy model can be applied through the six levels of the model adjusted to the goals and challenges of the teaming concept (Figure 6).

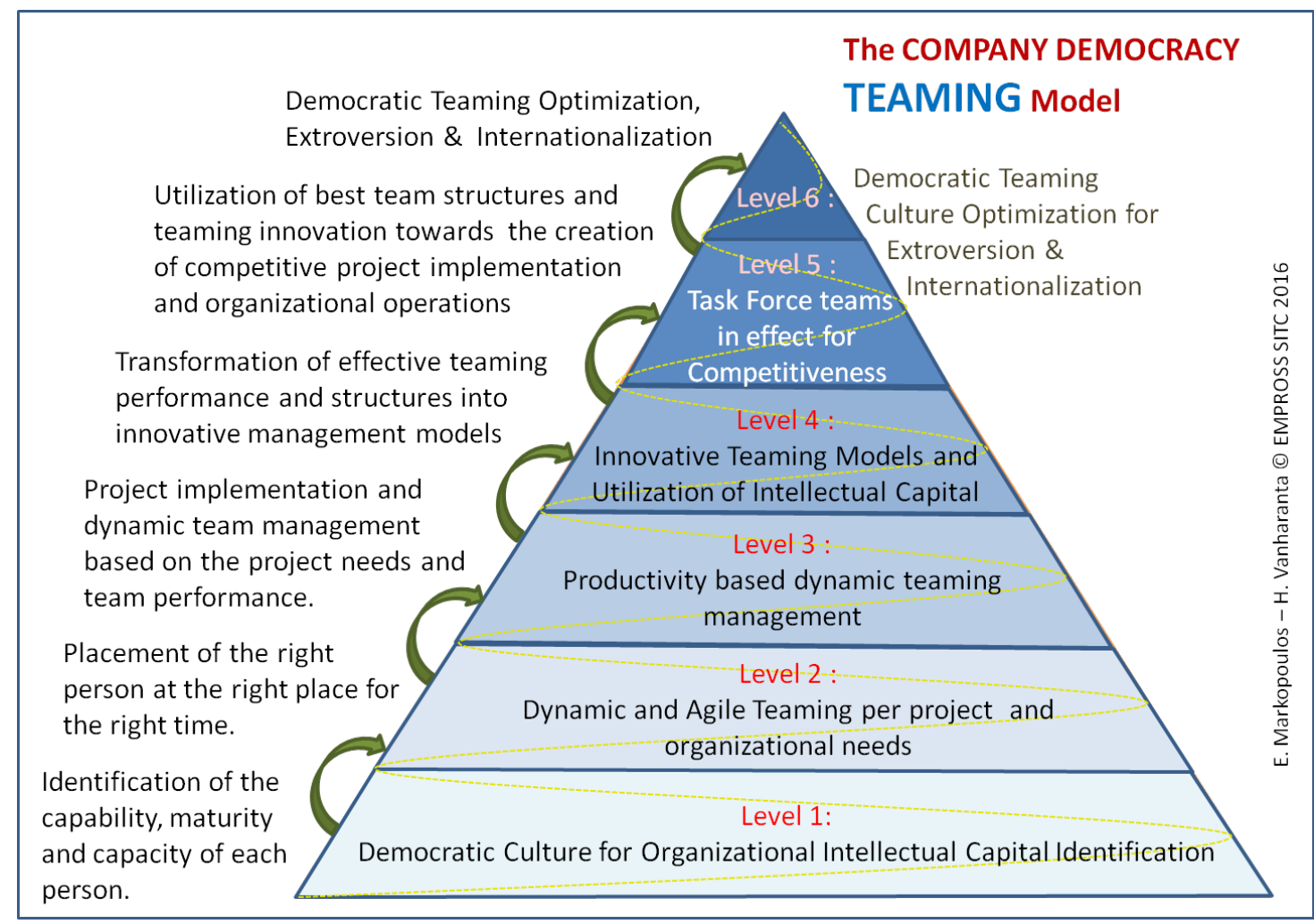

Figure 6. The company democracy model for teaming

The first level of the company democracy model for teaming creates a democratic organisational culture for knowledge elicitation, which is to be used in strategic development and leadership actions within an organisation. Democratic team 
management and leadership are the core engines for generating the wealth of information that constitutes and represents corporate knowledge, capability, competence and maturity, as well as the degree of democratic corporate management. It is where the democratic culture of speaking, sharing knowledge, seeking the opportunity, contributing to the organisation and much more takes place. Prior to anything else, the culture must exist if knowledge is to be generated via organisational self-awareness.

The second level of the model analyses and utilizes the information and knowledge generated from the first level, identifies the knowledge sources of the organisation, and places the right people in the right place at the right time. Level two actually works within a dynamic organisation chart. Nobody stays in a specific position unless there is a reason to stay there for a specific time. This means that a project manager cannot be a project manager on a project if it is beyond their areas of expertise, or that a manager in general cannot stay for long in a specific position unless and until productivity and efficiency results indicate that they should either remain, be removed or get promoted. To the same extent, employees with bright ideas and the willingness to work may be promoted to project manager without the typical credentials that the position requires because they have the desire and passion to work. In such cases, support can be allocated to help innovative minds carry out their vision. The overall idea of level two is to give all the people, who proved at level one that they have the knowledge and capability to provide added-value to the organisation, the opportunity to do so. The continuous results of everyone's work will determine their next position and the time they remain in that position.

The third level of the company democracy model promotes the measurements concerning the effectiveness of teams involved on the projects to which they have been allocated. It is the field where the ball is played and the arena where the ones who desired the opportunity are placed in order to prove that they are worth being given the chance they are looking for. The third level of the model is the actual application of the knowledge generated at level one and the judgment behind the management's decision at level two in forming teams based on that knowledge, rather than on the quantified project characteristics of each team member. The performance metrics at level three are in various dimensions, such as organisational, financial, productivity, profitability, performance, innovation, communication and production. 
The fourth level of the model identifies the innovation generated due to the effectiveness of the team during the implementation of a project. Good teams not only work well, but also have the capability and competence to innovate while they perform a given task by integrating their expertise, skills and team spirit. Well-structured teams, which operate under a democratic culture, are very likely to come up with innovative ways to overcome project challenges that sometimes can be much more than project innovation. Level four supports alternative project implementation practices to the teams at level three. The level documents alternative ways used by a team to execute a project, examines its added-value in terms of a shared value, as well as its financial, organisational and overall performance impact, and identifies the innovation produced on implementing project management.

The fifth level of the model transforms the innovation developed in level four into cooperative competitiveness for the organisation. This is the level at which the project execution innovations, of any kind, as defined at level four, become competitive advantages for the organisation's products, services or processes. Such advantages can be new management models, new management processes, new teams or groups of experts as special implementation teams, task force teams, conflict and resolution management teams, special support teams and others type of teams, which provide competitiveness to the organisation through innovative ways in which teams are generated and projects are executed and supported.

The sixth level of the model capitalizes on democratic teaming experience, expertise, innovations and competitiveness at an international level through an extroversion strategy. In this case, the organisation can develop highly competitive teams, which can work on critical and highly important international projects for the organisation, with other international partners with high management and operational capacity. The organisation can also export the knowledge and innovation created through the democratic teaming culture in the form of management consulting services to other organisations or international initiatives. Teams at this level of maturity are significant assets for any organisation's international strategy.

The six levels of the company democracy model for teaming are aligned with the original operational models of the company democracy model, thereby forming the evolution of democratic teaming (Figure 6). 


\section{Evolution of Democratic Teaming Culture}

In real life, it is actions, not words that count in modern management. Those who 'speak big' can have the opportunity to not only 'look big' but 'act big'. Democracy is a gentle tool and system, which distinguishes the ones seeking opportunities from the ones hiding from them. It is a system that promotes dialogue with the privileges and responsibilities that freedom comes with. Being truly free might not be what many people want, as they may prefer to be virtually free in a controlled environment where the freedom to speak and act is a theoretical concept, but nothing more. It is the bosses in a company, the governors in a country and the leaders in general who take the calls on behalf of those with the illusion that they are free to participate if they want to, even though they do not participate, either because they do not want to, because they cannot or because they are not allowed to.

The reality behind these situations is that people fear democracy, because most, if not all, of them have never lived in an actual democratic culture, where coexistence, coevolution and co-development were fostered under cooperation and not competition. A democratic teaming culture (Figure 7) can be seen as an applied philosophy project and initiative for an organisation. Without democracy, people cannot really be heard, judged fairly, defend their values and, therefore, be allocated to the right team at the right time in the right place for the right duration. 


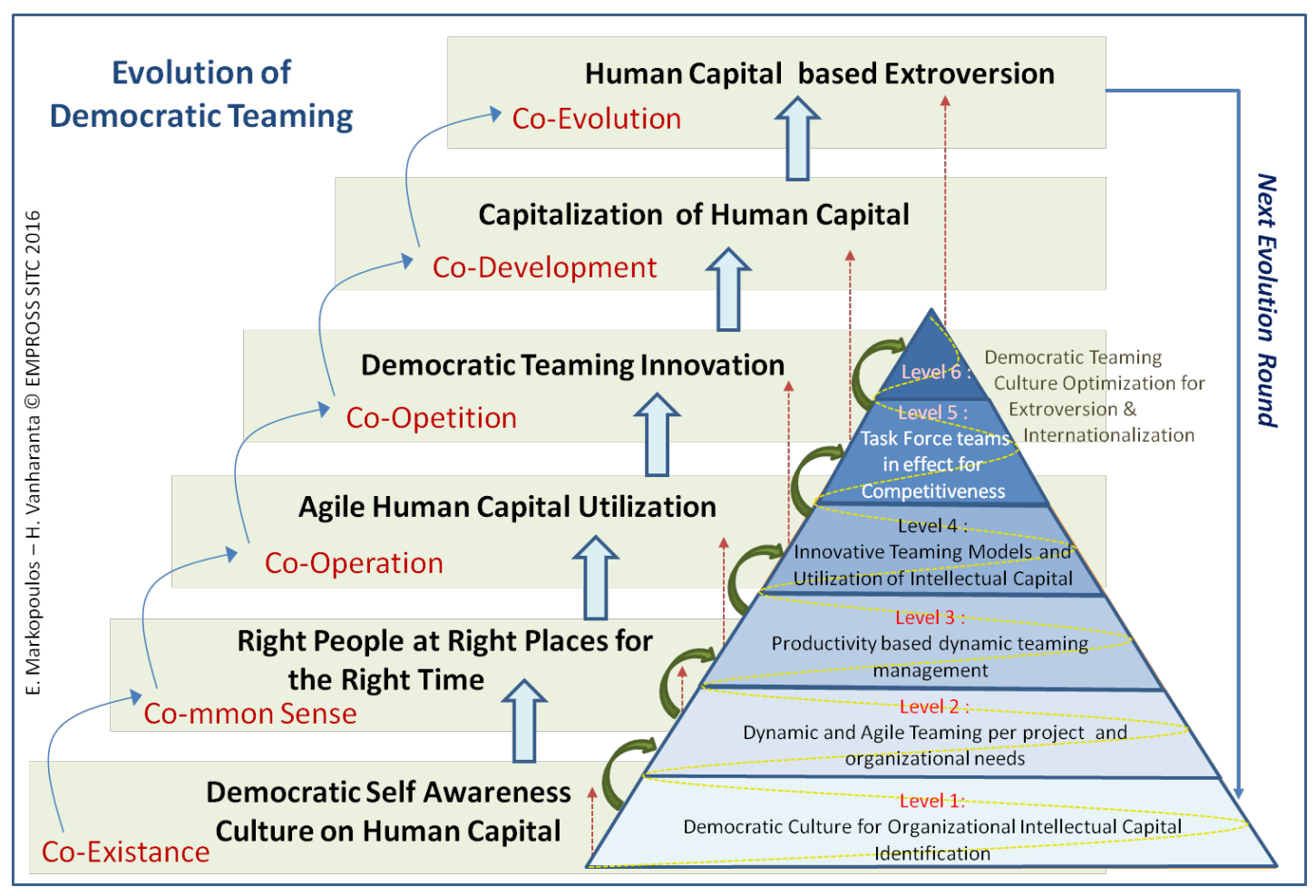

Figure 7. The evolutionary concepts of company democracy teaming

It is often stated that well-structured processes are essential for the effectiveness of organisational management. That said, for processes to be effective, they must be executed within an overall ethical framework in the context of an organisational culture. Processes that promote quantified discipline can certainly benefit an organisation by reducing mistakes and risks, but they also reduce democracy and the opportunities that come with it.

In this sense, teaming cannot be achieved by using structured processes, role descriptions, boxes, checklists and to-do lists. Effective teaming must involve the freedom that needed for everyone to feel that they belong on a team and not under the command of a boss. This highly critical and sensitive element when it comes to defining and managing freedom, not only in teaming but also in organisational management and leadership, must be approached in terms of an overall company democracy culture, which will embed such meanings in employees over time and lead to practical benefits for all.

\section{Democratic Teaming Management and Leadership}

Building and managing teams within a company democracy framework requires basic common sense, even if the approach seems quite revolutionary in relation to current 
management standards. The most important principle, besides the fundamental one, that teams are built to serve projects, as opposed to projects being served by established teams, is that no team member is permanent on any team. This also applies to the team itself, as no team can be permanent in an organisation, unless there is a need to exist, with specific work to do and quantified added-value for the organisation.

Today, in the age of slim or lean organisational management, agility, adjustability, dynamic operations and management are essential. Organisations must use all possible human resources at their disposal (employees, contractors, associates etc.) in order to find the best possible way of achieving their operational goals and strategy. Therefore, everyone who gets paid counts.

All employees are assets in a modern organisation and must be deployed based on their capacity, rather than simply according to their quantified and documented skills. Such capacity can also derive from the willingness that some employees have in accepting challenges: the very niche expertise they might have on a very specific subject, the loyalty to the organisation, the desire for the opportunity, the chance they are looking for to speak, innovate, be heard and be given the opportunity to prove their added-value.

As such, capacities cannot be quantified; it is not easy for the team builder to identify each person unless each person gets the opportunity to speak and operate in a democratic organisational culture, which sooner or later, usually sooner, will expose their skills, capacities and competence. Another democratic teaming management principle is to know what everyone can really do, rather than what everyone seems to be able to do, before building a team. Having done that, a team builder can build the right team for the right project in line with the three teaming dimensions: person, project and duration.

Each teaming dimension represents an axis on a three-dimensional Cartesian 3 system (Figure 8). The X-axis represents the 'right person', which takes an employee's capability, competence, maturity and capacity into account, while the ' $\mathrm{i}$ ' value is the employee's score. The Y-axis represents the 'right place', which takes the project value in terms of criticality and complexity into account, while the ' $\mathrm{j}$ ' value is the project's complexity score. The Z-axis represents the 'right time', which takes into respect the duration of the project, while the ' $\mathrm{k}$ ' value is the project time score. 
The value of a team member ' $A$ ' is located within the $P_{\text {erson }}(A)$ as $P A=\{i, j, k\}$ vector. The area that is covered by the PA vector is represented by the vector $A_{\text {rea }}(A)$, as A (A) $\{\mathrm{i}, \mathrm{j}, \mathrm{k}\}$.

The size of this area indicates the effectiveness of the person in a team. The larger the area, the more skilled and effective a team member can be and, in turn, the less likely to be replaced during the overall project implementation process.

The following phrase indicates that employee B is better placed (has a larger effectiveness value) on a team than employee A:

$$
\mathrm{A}_{\text {rea }}=>\mathrm{P}(\mathrm{A})\{\mathrm{I}, \mathrm{j}, \mathrm{k}\}=\mathrm{A}(\mathrm{A})\{\mathrm{I}, \mathrm{j}, \mathrm{k}\}<\mathrm{A}(\mathrm{B})\{\mathrm{I}, \mathrm{j}, \mathrm{k}\}=\mathrm{P}(\mathrm{B})\{\mathrm{I}, \mathrm{j}, \mathrm{k}\}
$$

The last dimension, which represents the duration of a team member's presence on a team, is the most important in the democratic teaming model. As projects, do not belong to employees, the same applies to their jobs, titles, positions and hierarchies, which also have no owner, other than the conceptual organisation. Placing the right person in the right position for too long is like giving that person the authority to own the position and manage it according to the interests, which can help to maintain it or lead to promotion. In many cases, this creates autarchic managers, who consider the area in which they work as their property. In the same way, keeping the right person in the right position for too short a period may not provide this person with enough opportunity to contribute their best and prove what they are capable of. 


\section{Democratic Teaming 3D Matrix for Team member Effectiveness}

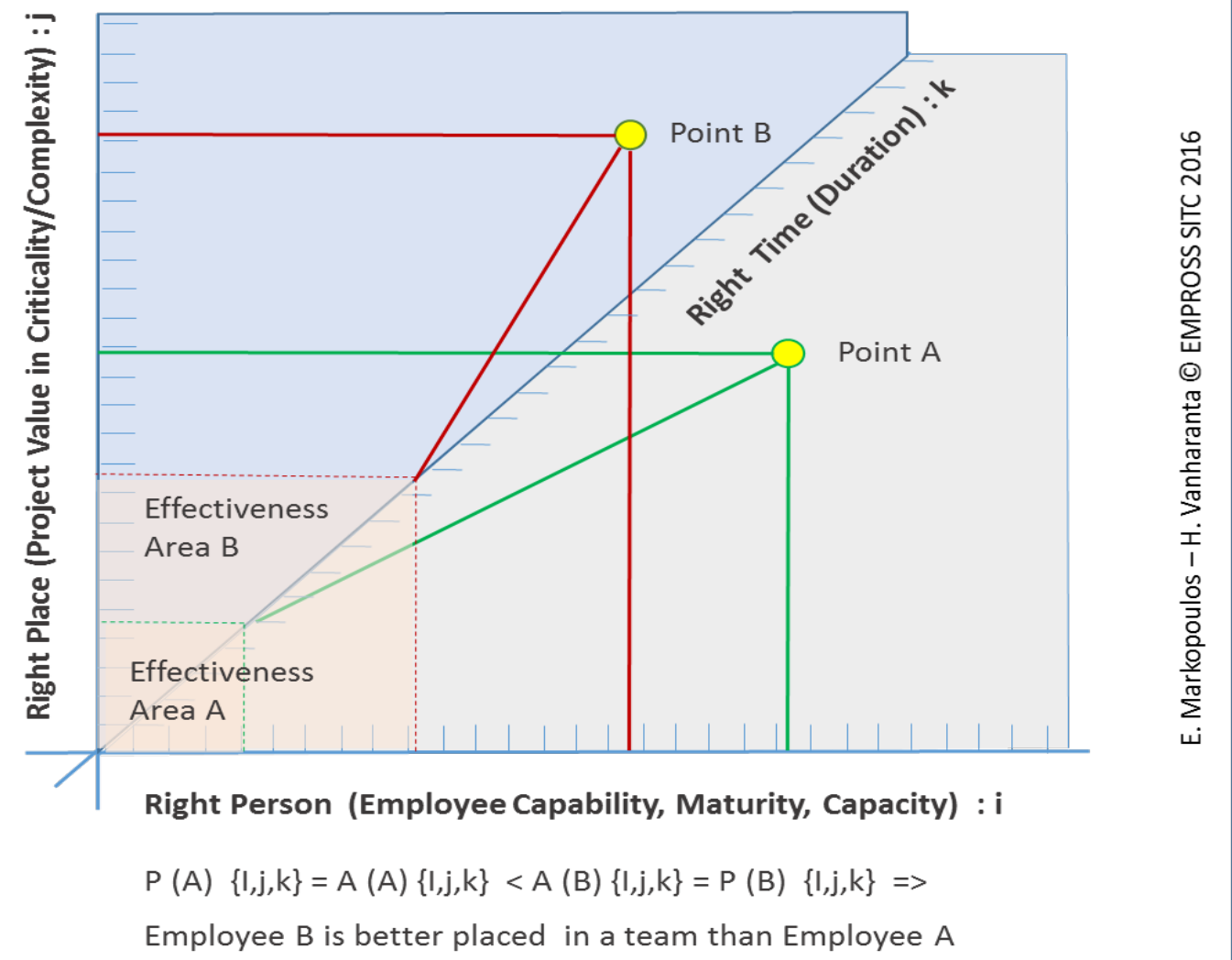

Figure 8. Democratic teaming dimension coordinates

The duration of the period in which the right person is in the right place is related to the work that needs to be done. A person can stay too long on a team or in a position if there is enough work to be done, if their expertise is not needed elsewhere or they perform with the same enthusiasm they displayed when first placed on that team or in that position. Likewise, spending too short a period of time on a team or in a particular position may result from an incapacity of the person to perform as expected, a lack of enthusiasm or the need to deploy their expertise elsewhere in the organisation.

This kind of organisational management and leadership approach, which eliminates static hierarchies in both positions and people occupying them, can only be achieved with an effective democratic teaming and organisational management, as well as an effective leadership framework and culture. As people, can benefit from a change, organisations can benefit from changing too. Democracy allows organisations to learn more about their human assets and manage them accordingly for the benefit of the organisation, which includes employees remaining in a successful organisation. 


\section{A Shared Added-Value in the Democratic Project Teaming}

The principles of the company democracy model in some cases might not be pleasant for people who desire the comfort to realize their career achievements through the positions they hold. The fact that the model promotes dynamic and agile organisational structures, with any permanent positions, titles or any other privilege for anyone without a specific reason and purpose and for a given time, could be considered to be somewhat radical in terms of the benefits offered to certain groups of employees, but also quite revolutionary and inspiring for other groups of employees.

Those who seek the opportunity, but are forced to stand aside due to their limited quantitative criteria, might regard the model as a great opportunity to pursue what their ambition. On the other hand, those who feel that they have worked hard enough in order to obtain a position, and thus have no intention to give back anything more, might feel a bit uncomfortable. The bottom line in this case is that the model is based on the philosophy that strong organisations lead to a strong economy, while strong economies lead to strong nations and societies through the wealth they produce and the employment they offer. As such, no employee in any organisation should be underutilized.

In order to achieve this organisational shared value, people must give back to the organisations employing them, while the organisations must give back to their people. Without a democratic culture in an organisation's management and leadership, this balance cannot be achieved. Organisations need all their employees to contribute to the development and stability that can assure such a return for the society. In the case of static and bureaucratic team and organisational management, with limited or no rotation at all, unless it is absolutely necessary, it is impossible for an organisation to first identify the capacity of its employees and then to use this effectively for the benefit of everyone.

The shared value of the company democracy model for teaming can be extended through the innovation and competitiveness that the model provides to the organisation and society. Under the philosophy of 'One Person, Many Insights', democratic teaming culture provides opportunities for more innovation, ideas, best practices, products and services to be derived, on account of the fact that more people have the chance to call the shots, or for those shots to be called by those who want the chance more than others. It is 
not surprising that many projects, ideas and investments fail due to a lack of commitment, enthusiasm or innovation. Such failures have a significant impact on the organisations that invest in them, as well as on society, in terms of not obtaining the desired outcomes. With the right people in the right place at the right time, the benefits reach everyone, even the ones who are complaining, because the democratic teaming model gives everyone the opportunity to give feedback.

\section{Conclusions}

Democracy is a word with a most contradictory usage. Everyone loves it, but many hate it when it comes to be applied in practice. Everyone wants to be democratic, listen to the people, provide opportunities, and share knowledge, ideas and insights, as long as their interests are not under threat. The company democracy model considers the people to represent the most significant and valuable resources and assets that any organisation has. The teaming version of the company democracy model makes sure that those assets are used wisely for the benefit of the organisation and society, as well as those who are part of the organisation. No employee is above the company and no one can own a company within a company by taking advantage of the authority that comes with their position.

Heraclitus, one of the most significant Greek philosophers, wrote in the first fragment of his work that, "Everything flows and nothing abides; everything gives way and nothing stays fixed" [21]. Nothing stays still, as the laws of nature are evolutionary and therefore dynamic. Teaming is a dynamic activity as well by bringing together the most suitable members at the right time to work on a specific project. It is an activity that must align the project elements with the elements of the human resources capacity for the benefit of the project and the organisation. As projects are systemic and dynamic, due to the evolution of the economy and society, teams must also be dynamic and agile of organisations are to utilize the best resources in order to create shared added-value [22]. Since there are no super heroes, the need for a co-evolution and coexistence management model in organisations is more than a necessity.

Democracy and the company democracy model represent tools for applying this coevolutionary, co-developmental and cooperative management and leadership philosophy. The company democracy teaming model applies this philosophy to the challenge of 
building teams for projects, not projects for teams. Organisations fail, not because they are unable to solve their problems, but because they cannot recognize them early enough. Identifying problems, on the other hand, requires agility and democracy if everyone is to contribute and be part of the solution, as opposed to staying silent and being part of the problem. Teams are built to solve problems and implement projects, initiative, strategies and visions. Democracy offers a unique opportunity for all to speak and be part of the solution, on the right team and at the right time.

The company democracy teaming model, based on an evolutionary teaming culture, is an applied management and leadership philosophy based on the Delphic maxims of 'gnothi seauton' (know thyself), 'metron ariston' (moderation is best) and 'miden agan' (nothing in excess) [16]. To build a team, self-assessment is important as it helps to understand the project requirements as opposed to personal capacity. It is also important for people to be modest, firstly respecting the project and then themselves through their contribution to the project. It is also vital for nothing to be in excess, driven by personal benefits and vain egocentrism. Such values need to be applied to team building in order to ensure organisational and personal success, as well as concurrently generate a shared value with society and humanity.

The use of applied philosophy in relation to team building and teaming in a project context, via democratic management and leadership processes, is an alternative way to best utilize the human capital in an organisation. Although the company democracy teaming model emanates from applied philosophy, it is probably also common sense to pursue a common goal, despite the fact that common sense does not seem to that common.

\section{References}

1. Markopoulos, E. \& Vanharanta, H., 2016. Space for Company Democracy, Proceedings of the International Conference on Applied Human Factors and Ergonomics AHFE 2016, Orlando Florida, USA.

2. Markopoulos, E. \& Vanharanta, H., 2015. The Company Democracy Model for the Development of Intellectual Human Capitalism for Shared Value, Elsevier, Procedia Manufacturing, Volume 3, pp. 603-610.

3. Robbins, S., 2016. Build Your Management Team, Entrepreneur, Sited at 17 July, 2016, Available at: https://www.entrepreneur.com/article/83618.

4. Harvard Business School, 2016. The Importance of Teaming. Harvard Business School Working Knowledge Baker Library, Bloomberg Center. Sited at 17 July, 2016, Available at: http://hbswk.hbs.edu/item/the-importance-of-teaming. 
5. Jackson, M., C., 2003. Systems Thinking: Creative Holism for Managers, John Wily \& Sons, Ltd, England.

6. Edmondson, A., 2012. Teaming: How Organizations Learn, Innovate, and Compete in the Knowledge Economy, Wiley \& Sons.

7. Miller, J. 1978. Living systems. New York, McGrew-Hill. 1102 p.

8. Samuelson, K., 1978. General information systems theory in design, modelling and development. Information science in action, Systems Design. Vol. 1. NATO Advanced Study Institute 59 in Crete August 1978. Nijhoff, Boston. pp. 304-320.

9. Samuelson, K., 1978. General information systems theory in design, modelling and development. Information science in action, Systems Design. Vol. 1. NATO Advanced Study Institute 59 in Crete August 1978. Nijhoff, Boston. pp. 304-320.

10. Österlund, J., 1994. Competence Management by Informatics Systems in R\&D

Work. Stockholm, Royal Institute of Technology. 205 p.

11. Yackinous, W., S., 2015. Understanding Complex Ecosystem Dynamics: A Systems and Engineering Perspective. Elsevier.

12. Edmondson A. 2013, The Three Pillars of a Teaming Culture of Teaming. Harvard Business Review. Sited at 17 July, 2016, Available at: https://hbr.org/2013/12/thethree-pillars-of-a-teaming-culture/

13. Slater, P. \& Bennis, W., 1990. Democracy is Inevitable. Harvard Business Review. Vol. 68. pp. 167-176.

14. Markopoulos, E. \& Vanharanta, H. 2014. Democratic Culture Paradigm for Organizational Management and Leadership Strategies - The Company Democracy Model, Proceedings of the 5th International Conference on Applied Human Factors and Ergonomics AHFE 2014, Krakow, Poland.

15. Vanharanta, H. \& Markopoulos, E. 2013. Creating a Dynamic Democratic Company Culture for Leadership, Innovation and Competitiveness. 3rd Hellenic-Russian Forum. September 17, Athens, Greece.

16. Parke H. \& Wormell, D. 1956. The Delphic Oracle, Basil Blackwell, vol. 1, p. 389.

17. Kantola, J., Vanharanta, H. \& Karwowski, W. 2006. The Evolute System: A CoEvolutionary Human Resource Development Methodology. The International Encyclopedia of Ergonomics and Human Factors. Karwowski, W., CRC Press, Boca Raton, Florida, USA.

18. Paajanen, P., Piirto, A., Kantola, J. \& Vanharanta, H. 2006. FOLIUM - Ontology for Organizational Knowledge Creation. 10th World multi-conference on systemics, cybernetics and informatics. Orlando, Florida, USA.

19. Nonaka, I. \& Takeuchi, H. 1995. The Knowledge-Creating Company: How Japanese Companies Create the Dynamics of Innovation, New York, Oxford University Press,p. 284. 
20. Butler-Bowdon, T., 2013. 50 Philosophy Classics: Thinking, Being, Acting, Seeing, Nicholas Brealey Publishing.

21. Salo, M., Markopoulos E., Vanharanta H. \& Kantola, J. 2016. Degree of Agility with an Ontology Based Application. Proceedings of the 7th International Conference of Applied Human Factors and Ergonomics (AHFE) at the Conference Proceeding, Orlando Florida, USA 